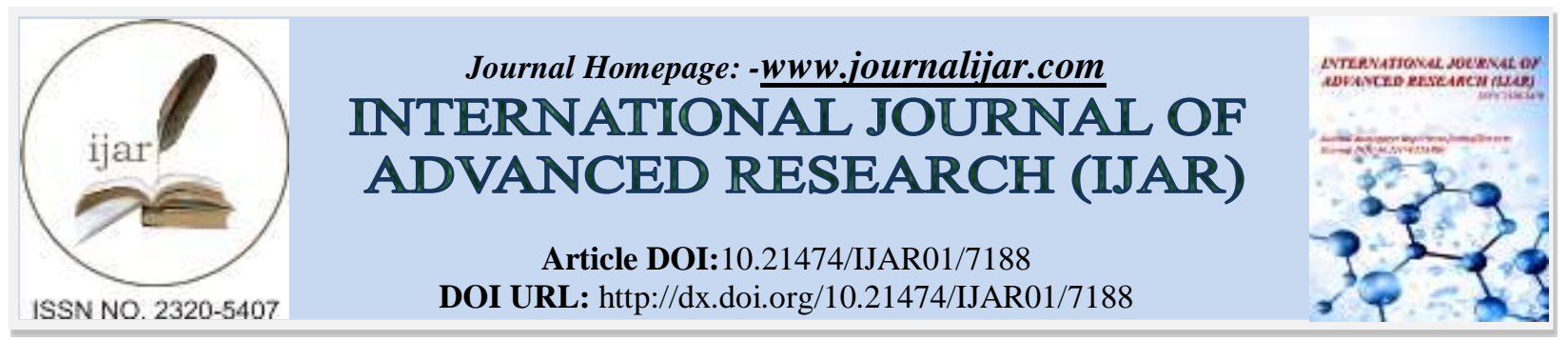

RESEARCH ARTICLE

\title{
ANTIMICROBIAL ACTIVITY OF ALLIUM CEPA EXTRACTS AGAINST AEROMONASHYDROPHILA ISOLATED FROM DIARRHEA SAMPLES OF CHILDREN IN IRAQ.
}

\author{
Ban mahdi shakir ${ }^{1}$, Sinaamahdi shakir ${ }^{2}$, Nadhee mahammood hussein ${ }^{3}$ and khetam habeeb rasool ${ }^{3}$. \\ 1. Imam Sadiq General Hospital, Babil Health Directorate /Ministry of Health /Iraq. \\ 2. Teaching Laboratories Medical City/Ministry of Health /Iraq. \\ 3. Department of Biology, College of Science, Al-Mustansiryah University.
}

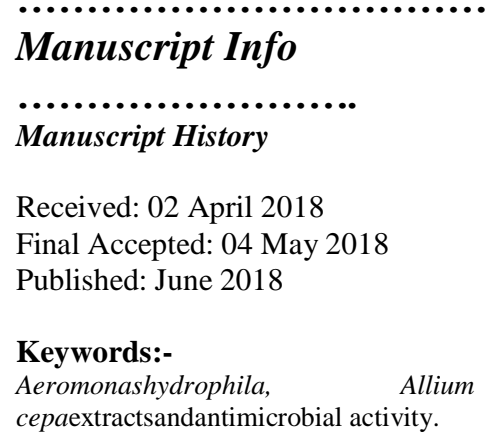

Aeromonashydrophila, Alliu
cepaextractsandantimicrobial activity.

\begin{abstract}
Two hundred and seventy five watery diarrhea samples (clinical samples) were taken from children suffering from gastroenteritis. Twenty isolates of Aeromonashydrophila (7.2\%)were obtained.The organisms were identified as Aeromonashydrophila according to a biochemical tests and also by API $20 \mathrm{E}$ system.

Among the infected patients the age ranged from less than 1 month to 12 years. The highest incidence of Aeromonashydrophilaisolation was among(less than 1 month -2) years old age group $16(80 \%)$ isolates.

The antibiotic-sensitivityof Aeromonashydrophila isolates was determined to thirteen different antibiotic discs. All isolates were resistant $(100 \%)$ to amoxicillin, while all the isolates were sensitive $(100 \%)$ to ciprofloxacin, norfloxacin, chloramphenicol, tetracycline, Imipenem, cefotaxime and cefteriaxon.

Antimicrobial activity of the Allium cepa (white and purple onion) extracts was tested against Aeromonashydrophila isolates was done. Among the three types of white onion extracts tested, cold-water, hot water and ethanolic extracts, only hot- water extract showed antibacterial activity. On the other handpurple onion extracts did not show any inhibitory effect on the growth of Aeromonashydrophila isolates.
\end{abstract}

Copy Right, IJAR, 2018,. All rights reserved.

\section{Introduction:-}

The genus Aeromonas represents a group of gram negative facultative anaerobic bacteria.They are oxidase positive organisms and motile organisms. They can be isolated from many environmental location, but they are mainly water- born organisms found in virtually all waters, including chlorinated drinking water(1).Aeromonashydrophila produce several toxins including haemolycin, and enterotoxins and a repertoire of enzymes which digest cellular components, such as proteases, amylases, and lipases (2).

Motile aeromonads have been reported to cause a wide range of diseases in both man and animals. Human aeromonas infections include skin and soft tissue infections, gastroenteritis, and bacteremia. Gasroenteritis is the most common human illness associated with Aeromonashydrophila. It mainly affecting the young, the elderly and immunocompromised patients (1). 
Antibiotics resistant microorganism became a major clinical and public health problem during the lifetime of most people. This shift in susceptibility greatly affects our ability to successfully treat patients empirically. Plant derived products have been used for medicinal purposes for centuries. At present, it is estimated that about $80 \%$ of the world population rely on botanical preparations as medicines to meet their health needs. Herbs and spices are generally considered safe and proved to be effective against certain ailments. They were also extensively used, particularly, in many Asian, African and other countries. In recent years, in view of their beneficial effects, use of spices/herbs has been gradually increasing in developed countries also (3).

Search for new antimicrobials is very important in recent times, considering the escalating levels of antibiotic resistance among pathogenic bacteria. So this study aimed to isolation of Aeromonashydrophila from acute gastroenteritis in children, study the susceptibility of Aeromonashydrophila to defferent antibiotics and study the antimicrobial activity of Allium cepa (onion) extracts on Aeromonashydrophila by agar diffusion method.

\section{Materials and methods:-}

\section{Collection of Samples:-}

Two hundred and seventy five watery diarrhea samples (clinical samples) were taken from children suffering from gastroenteritis collected from Welfare teaching hospital / Medical city. The samples were collected in sterile containers.

\section{Isolation of Aeromonas:-}

The clinical samples were inoculated in alkaline peptone water as enrichment medium and incubated for $24 \mathrm{~h}$ at $37^{\circ} \mathrm{C}$, one loopful of culture was inoculated on selective media (TCBS agar and MacConkey's agar plates) and incubated for $24 \mathrm{~h}$ at $37^{\circ} \mathrm{C}(4)$.

\section{Identification of Aeromonashydrophila:-}

Morphological and biochemical tests were applied for the bacterial isolates according to Forbes et al. (5) and Ciprian (6).Aeromonashydrophilaisolates were also identified to species level by using API 20 E (Analytic Profile Index) system (Bio-Merieux, France), which is a standardized system for the identification of bacteria belong to Enterobacteriaceae family.

\section{Antimicrobial Susceptibility Test:-}

Kirby-Bauer method was used as described by Baron and Finegold (7) to carry out the antimicrobial susceptibility test. Bacterial culture for 18-24 h. was compared with the standard turbidity solution (McFarland), this approximately equals to $\left(1.5 \times 10^{8}\right) \mathrm{CFU} / \mathrm{ml}$. A $0.1 \mathrm{ml}$ of the culture was spreaded on the surface of Mueller-Hinton agar plates, left to dry for 15 minutes at room temperature. Thirteen antibiotics used in this study (table-1).

The antibiotic discs were placed on the surface of the medium and incubated at $37^{\circ} \mathrm{C}$ for 24 h. Inhibition zones around the discs were measured according to National Committee for Clinical Laboratory Standards (NCCLS) (8).

Table 1:-Antibiotic discs used in this study

\begin{tabular}{|c|l|c|c|r|}
\hline \multicolumn{1}{|c|}{ Antibiotic name } & Symbol & Disk potency & Company (origin) \\
\hline $\mathbf{1}$ & Amoxicillin & AMX & $25 \mu \mathrm{g}$ & Bioanalyse(Turkyi) \\
\hline $\mathbf{2}$ & Amoxicillin-Na & AMX-Na & $10 \mu \mathrm{g}$ & Bioanalyse(Turkyi) \\
\hline $\mathbf{3}$ & Ampicillin & Amp & $10 \mu \mathrm{g}$ & Bioanalyse(Turkyi) \\
\hline $\mathbf{4}$ & Cefotaxime & CTX & $30 \mu \mathrm{g}$ & Bioanalyse(Turkyi) \\
\hline $\mathbf{5}$ & Cefterixon & CRO & $30 \mu \mathrm{g}$ & Bioanalyse(Turkyi) \\
\hline $\mathbf{6}$ & Cephalothin & CF & $30 \mu \mathrm{g}$ & Bioanalyse(Turkyi) \\
\hline $\mathbf{7}$ & Chloramphenicol & $\mathrm{C}$ & $30 \mu \mathrm{g}$ & Bioanalyse(Turkyi) \\
\hline $\mathbf{8}$ & Ciprofloxacin & Cip & 5 & Bioanalyse(Turkyi) \\
\hline $\mathbf{9}$ & Erythromycin & E & $15 \mu \mathrm{g}$ & Bioanalyse(Turkyi) \\
\hline $\mathbf{1 0}$ & Imipenem & Imp & $10 \mu \mathrm{g}$ & Bioanalyse(Turkyi) \\
\hline $\mathbf{1 1}$ & Norfloxacin & Nor & $10 \mu \mathrm{g}$ & Bioanalyse(Turkyi) \\
\hline $\mathbf{1 2}$ & Tetracycline & TE & $30 \mu \mathrm{g}$ & Bioanalyse(Turkyi) \\
\hline $\mathbf{1 3}$ & Trimethoprime & Tri & $25 \mu \mathrm{g}$ & \\
\hline
\end{tabular}




\section{Extraction of the plant materials(Onionextraction):-}

Two types of onion bulbs (Allium cepa) white and purple skinned were purchased from local markets in Baghdad, Iraq (figure-1). The onions were washed with clean sterile distilled water and allowed to air dry for one hour. The outer covering of the onion were manually peeled off. The onion bulbs being separated were washed and extracted in the following ways:

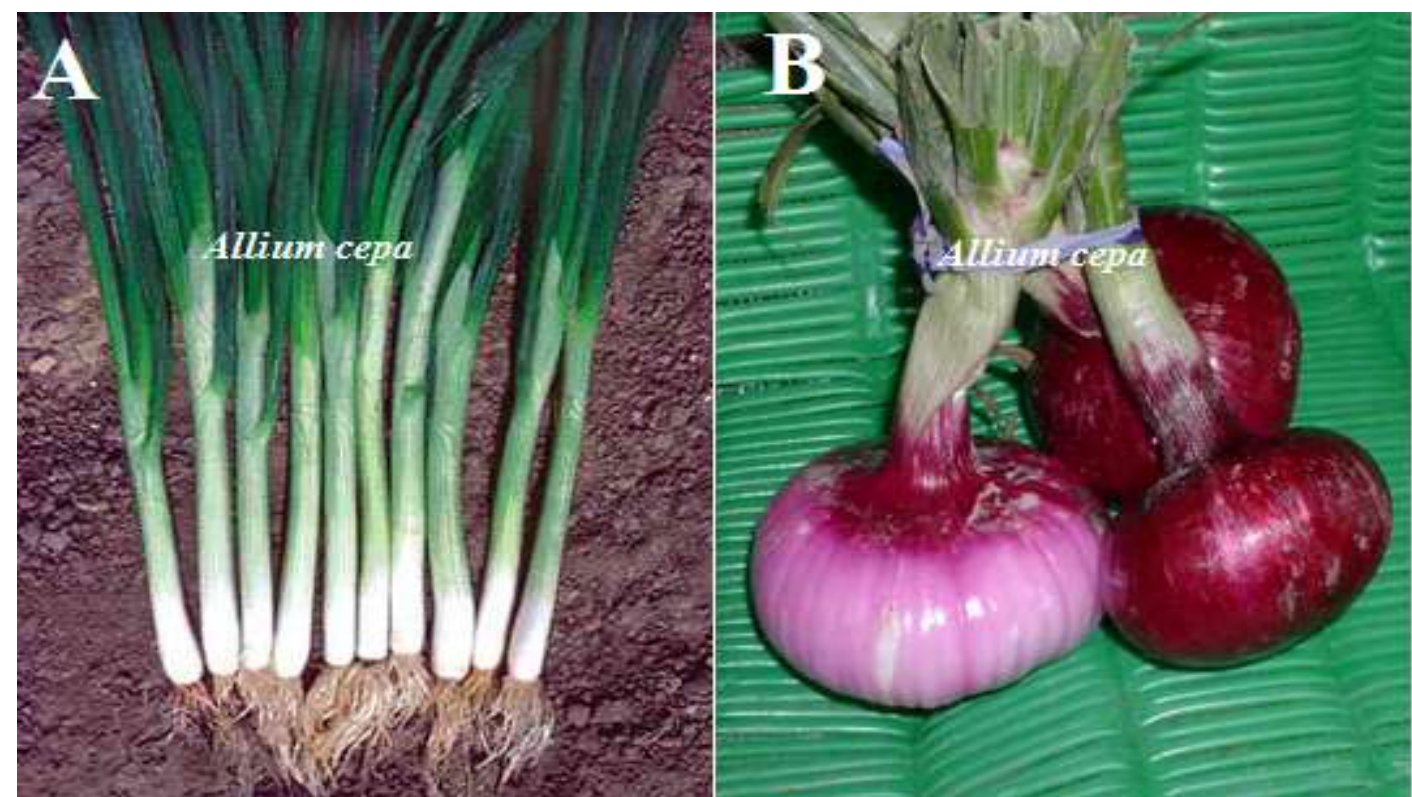

Figure-1:-Allium cepa (Onion); A: white skinned and B: purple skinned Onion

Exactly $200 \mathrm{~g}$ of fresh onion bulbs were blended into fine powder and soaked in $100 \mathrm{ml}$ of distilled water for $24 \mathrm{hr}$. The pulp obtained was left in a clean, sterile glass container and shaken vigorously to allow for proper extraction and it was filtered using a sterile muslin cloth after which the extract was obtained, air-dried and stored below ambient temperature until required. Exactly $200 \mathrm{~g}$ of fresh onion bulbs were blended and soaked in $100 \mathrm{ml}$ of hot water for $24 \mathrm{hr}$., the resultant juice was extracted, air-dried and stored as in above. Exactly $200 \mathrm{~g}$ of fresh onion bulbs were blended and soaked in $100 \mathrm{mls}$ of $95 \%$ ethanol for $24 \mathrm{hr}$ and the extract was obtained, air-dried and stored as in above (9).

Innoculum Preparation by Direct Colony Suspension Method;-

A small volume of sterile water was poured inside a test tube to which general colonies of the test organisms, taken directly from the plate were emulsified and the suspension was adjusted to match the 0.5 McFarland's standard this approximately equals to $\left(1.5 \times 10^{8}\right) \mathrm{CFU} / \mathrm{ml}$, which has a similar appearance of an overnight broth culture by adding distilled water (9).

Antimicrobial activity of onion extracts against Aeromonashydrophila isolates:-

The sensitivity test ofAeromonashydrophila to the extracts of Allium cepa (onions) was carried out using the cupplate diffusion method. A glass dropper was used to add $0.02 \mathrm{ml}$ of the suspension to an already prepared medium. A sterile cotton swab was used to spread by streaking the organisms all over the surface of the medium and allowed to dry for about 5 minutes. Cups of $6 \mathrm{~mm}$ in diameter were made in the agar using sterile corkborer.

Different dilutions of the plant extracts prepared in the order of $100 \mu \mathrm{g} / \mathrm{ml}, 200 \mu \mathrm{g} / \mathrm{ml}, 300 \mu \mathrm{g} / \mathrm{ml}, 400 \mu \mathrm{g} / \mathrm{ml}$ and $500 \mu \mathrm{g} / \mathrm{ml}$ respectively were prepared in five different test tubes and placed in a test tube rack. Exactly $0.02 \mathrm{ml}$ of each concentration was introduced into each hole on the medium and was allowed to stand on the bench for about one hour for proper diffusion. It was thereafter incubated at $37^{\circ} \mathrm{C}$ for $24 \mathrm{hr}$. The sensitive bacteria grew everywhere except in areas around the holes in the medium. Then, the resulting inhibition zones obtained were measured in millimeters and recorded against the corresponding concentrations (9). 


\section{Results and Discussion:-}

Isolation and Identification of Aeromonashydrophila:-

Two hundred and seventy five watery diarrhea samples were collected from patients suffering from gastroenteritis children fromless than 1 month to12 years of age.

In this study, alkaline peptone water was used as an enrichment medium for isolation of Aeromonas, Robinson et al. (10) and kayet al. (11) reported that this medium yielded 2.6 times the number of isolates compared to other media and was recommended for the isolation of Aeromonasfrom humans.

The isolates that were obtained from the selective media were identified according to Forbes et al. (5) and Ciprian (6).All isolates were grown on MacConkey's agar and TCBS agar with pale yellowish, and yellow color colonies, respectively.All isolates were also identified by Api-20E system as shown in table- 2 .

Table 2:-The results of Api-20 E system

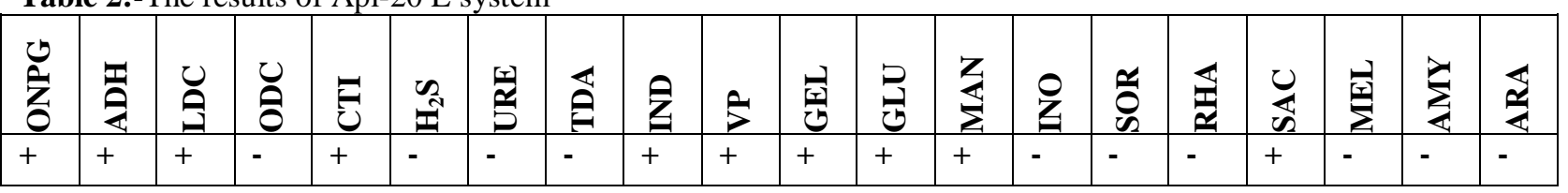

Distribution of Aeromonashydrophilaamong infected patientsaccording toage:-

Out of 275 watery diarrhea samples, 20(7.27\%)Aeromonashydrophila isolates were obtained.The distribution of Aeromonashydrophilaaccording to age of infected patients was summarized in table-3. Among the infected patients the age ranged from less than 1 month to 12 years. The highest incidence of Aeromonashydrophilaisolation was among(less than 1 month -2) years old age group $16(80 \%)$ isolates. This may due to contamination of water and food, malnutrition and immune deficiency of children in compare with adults.

Lower proportions of isolation were obtained by Aslani and Alikhani,(12); Juan et al., (13) with 3.4\% and 2.3\%, on the other hand, Kannanet al. (14) and Obi and Bessong (15) found higher proportions of isolation with $10.6 \%$ and $13.3 \%$.

Table -3:-Distribution of Aeromonashydrophilaisolates according to age

\begin{tabular}{|c|c|c|}
\hline $\begin{array}{c}\text { Patient age } \\
\text { (Years) }\end{array}$ & No. of cases evaluated & $\begin{array}{c}\text { No. of } \\
\text { Aeromonashydrophila positive cases }\end{array}$ \\
\hline Less than 1 month -2 & 218 & 16 \\
\hline $2-4$ & 36 & - \\
\hline $4-6$ & 5 & 3 \\
\hline $6-8$ & 4 & - \\
\hline $8-10$ & 4 & - \\
\hline $10-12$ & 8 & 1 \\
\hline Total & 275 & 20 \\
\hline
\end{tabular}

\section{Antimicrobial Susceptibility Testing results (Disk Diffusion Method):-}

Thirteen different antibiotic discs were used to perform this test, along with all Aeromonashydrophila isolates. Results are shown in table -4 .

The results showed that allAeromonashydrophilaisolates (100\%) (20 isolates) were resistant to amoxicillin, 90\% (18 isolates) to cephalothin and 75\% (15 isolates) to erythromycin. However, 25\% (5 isolates) were resistant to trimethoprime. Resistance to Ampicillin was found to be 95\% (19 isolates). Moreover, 30\% (6 isolates) were able to resist amoxicillin-Naladixic acid. All isolates were sensitive100\% to, ciprofloxacin, norfloxacin, chloramphenicol, tetracycline, Imipenem, cefotaxime and cefteriaxon .

In case of cephalothin, $90 \%$ of the isolates were resistant to this antibiotic. The higher number of resistance exhibited by these bacteria was in good agreement with the result reported by Ram et al. (16). 
An encouragement result was found with norfloxacin, none of the isolates showed resistance to this antibiotic. Norfloxacin should be considered the drug of choice in the treatment of severe diarrhea in patients (17). Also none of our isolates were resistant to tetracycline, nitrofurantion and chloramphenicol.

Table-4:-Results of Suceptibility Test by using 13 Antibiotics Against Aeromonashydrophila isolates

\begin{tabular}{|c|c|c|c|c|c|c|c|c|c|c|c|c|c|}
\hline $\begin{array}{c}\text { No. of } \\
\text { isolate }\end{array}$ & Amp & AMX & $\begin{array}{c}\text { AMX- } \\
\text { Na }\end{array}$ & Cip & Imp & CTX & CRO & CF & TE & C & Nor & Tri & E \\
\hline $\mathbf{1}$ & $\mathbf{R}$ & $\mathbf{R}$ & $\mathbf{S}$ & $\mathbf{S}$ & $\mathbf{S}$ & $\mathbf{S}$ & $\mathbf{S}$ & $\mathbf{R}$ & $\mathbf{S}$ & $\mathbf{S}$ & $\mathbf{S}$ & $\mathbf{S}$ & $\mathbf{S}$ \\
\hline $\mathbf{2}$ & $\mathbf{R}$ & $\mathbf{R}$ & $\mathbf{S}$ & $\mathbf{S}$ & $\mathbf{S}$ & $\mathbf{S}$ & $\mathbf{S}$ & $\mathbf{R}$ & $\mathbf{S}$ & $\mathbf{S}$ & $\mathbf{S}$ & $\mathbf{S}$ & $\mathbf{R}$ \\
\hline $\mathbf{3}$ & $\mathbf{R}$ & $\mathbf{R}$ & $\mathbf{S}$ & $\mathbf{S}$ & $\mathbf{S}$ & $\mathbf{S}$ & $\mathbf{S}$ & $\mathbf{R}$ & $\mathbf{S}$ & $\mathbf{S}$ & $\mathbf{S}$ & $\mathbf{S}$ & $\mathbf{R}$ \\
\hline $\mathbf{4}$ & $\mathbf{R}$ & $\mathbf{R}$ & $\mathbf{S}$ & $\mathbf{S}$ & $\mathbf{S}$ & $\mathbf{S}$ & $\mathbf{S}$ & $\mathbf{R}$ & $\mathbf{S}$ & $\mathbf{S}$ & $\mathbf{S}$ & $\mathbf{S}$ & $\mathbf{R}$ \\
\hline $\mathbf{5}$ & $\mathbf{R}$ & $\mathbf{R}$ & $\mathbf{S}$ & $\mathbf{S}$ & $\mathbf{S}$ & $\mathbf{S}$ & $\mathbf{S}$ & $\mathbf{R}$ & $\mathbf{S}$ & $\mathbf{S}$ & $\mathbf{S}$ & $\mathbf{R}$ & $\mathbf{R}$ \\
\hline $\mathbf{6}$ & $\mathbf{R}$ & $\mathbf{R}$ & $\mathbf{S}$ & $\mathbf{S}$ & $\mathbf{S}$ & $\mathbf{S}$ & $\mathbf{S}$ & $\mathbf{R}$ & $\mathbf{S}$ & $\mathbf{S}$ & $\mathbf{S}$ & $\mathbf{S}$ & $\mathbf{S}$ \\
\hline $\mathbf{7}$ & $\mathbf{R}$ & $\mathbf{R}$ & $\mathbf{R}$ & $\mathbf{S}$ & $\mathbf{S}$ & $\mathbf{S}$ & $\mathbf{S}$ & $\mathbf{R}$ & $\mathbf{S}$ & $\mathbf{S}$ & $\mathbf{S}$ & $\mathbf{R}$ & $\mathbf{R}$ \\
\hline $\mathbf{8}$ & $\mathbf{R}$ & $\mathbf{R}$ & $\mathbf{R}$ & $\mathbf{S}$ & $\mathbf{S}$ & $\mathbf{S}$ & $\mathbf{S}$ & $\mathbf{R}$ & $\mathbf{S}$ & $\mathbf{S}$ & $\mathbf{S}$ & $\mathbf{R}$ & $\mathbf{R}$ \\
\hline $\mathbf{9}$ & $\mathbf{R}$ & $\mathbf{R}$ & $\mathbf{S}$ & $\mathbf{S}$ & $\mathbf{S}$ & $\mathbf{S}$ & $\mathbf{S}$ & $\mathbf{S}$ & $\mathbf{S}$ & $\mathbf{S}$ & $\mathbf{S}$ & $\mathbf{S}$ & $\mathbf{S}$ \\
\hline $\mathbf{1 0}$ & $\mathbf{R}$ & $\mathbf{R}$ & $\mathbf{S}$ & $\mathbf{S}$ & $\mathbf{S}$ & $\mathbf{S}$ & $\mathbf{S}$ & $\mathbf{R}$ & $\mathbf{S}$ & $\mathbf{S}$ & $\mathbf{S}$ & $\mathbf{S}$ & $\mathbf{R}$ \\
\hline $\mathbf{1 1}$ & $\mathbf{S}$ & $\mathbf{R}$ & $\mathbf{S}$ & $\mathbf{S}$ & $\mathbf{S}$ & $\mathbf{S}$ & $\mathbf{S}$ & $\mathbf{S}$ & $\mathbf{S}$ & $\mathbf{S}$ & $\mathbf{S}$ & $\mathbf{S}$ & $\mathbf{S}$ \\
\hline $\mathbf{1 2}$ & $\mathbf{R}$ & $\mathbf{R}$ & $\mathbf{R}$ & $\mathbf{S}$ & $\mathbf{S}$ & $\mathbf{S}$ & $\mathbf{S}$ & $\mathbf{R}$ & $\mathbf{S}$ & $\mathbf{S}$ & $\mathbf{S}$ & $\mathbf{S}$ & $\mathbf{S}$ \\
\hline $\mathbf{1 3}$ & $\mathbf{R}$ & $\mathbf{R}$ & $\mathbf{S}$ & $\mathbf{S}$ & $\mathbf{S}$ & $\mathbf{S}$ & $\mathbf{S}$ & $\mathbf{R}$ & $\mathbf{S}$ & $\mathbf{S}$ & $\mathbf{S}$ & $\mathbf{S}$ & $\mathbf{R}$ \\
\hline $\mathbf{1 4}$ & $\mathbf{R}$ & $\mathbf{R}$ & $\mathbf{R}$ & $\mathbf{S}$ & $\mathbf{S}$ & $\mathbf{S}$ & $\mathbf{S}$ & $\mathbf{R}$ & $\mathbf{S}$ & $\mathbf{S}$ & $\mathbf{S}$ & $\mathbf{R}$ & $\mathbf{R}$ \\
\hline $\mathbf{1 5}$ & $\mathbf{R}$ & $\mathbf{R}$ & $\mathbf{R}$ & $\mathbf{S}$ & $\mathbf{S}$ & $\mathbf{S}$ & $\mathbf{S}$ & $\mathbf{R}$ & $\mathbf{S}$ & $\mathbf{S}$ & $\mathbf{S}$ & $\mathbf{S}$ & $\mathbf{R}$ \\
\hline $\mathbf{1 6}$ & $\mathbf{R}$ & $\mathbf{R}$ & $\mathbf{S}$ & $\mathbf{S}$ & $\mathbf{S}$ & $\mathbf{S}$ & $\mathbf{S}$ & $\mathbf{R}$ & $\mathbf{S}$ & $\mathbf{S}$ & $\mathbf{S}$ & $\mathbf{S}$ & $\mathbf{R}$ \\
\hline $\mathbf{1 7}$ & $\mathbf{R}$ & $\mathbf{R}$ & $\mathbf{S}$ & $\mathbf{S}$ & $\mathbf{S}$ & $\mathbf{S}$ & $\mathbf{S}$ & $\mathbf{R}$ & $\mathbf{S}$ & $\mathbf{S}$ & $\mathbf{S}$ & $\mathbf{S}$ & $\mathbf{R}$ \\
\hline $\mathbf{1 8}$ & $\mathbf{R}$ & $\mathbf{R}$ & $\mathbf{R}$ & $\mathbf{S}$ & $\mathbf{S}$ & $\mathbf{S}$ & $\mathbf{S}$ & $\mathbf{R}$ & $\mathbf{S}$ & $\mathbf{S}$ & $\mathbf{S}$ & $\mathbf{R}$ & $\mathbf{R}$ \\
\hline $\mathbf{1 9}$ & $\mathbf{R}$ & $\mathbf{R}$ & $\mathbf{S}$ & $\mathbf{S}$ & $\mathbf{S}$ & $\mathbf{S}$ & $\mathbf{S}$ & $\mathbf{R}$ & $\mathbf{S}$ & $\mathbf{S}$ & $\mathbf{S}$ & $\mathbf{S}$ & $\mathbf{R}$ \\
\hline $\mathbf{2 0}$ & $\mathbf{R}$ & $\mathbf{R}$ & $\mathbf{S}$ & $\mathbf{S}$ & $\mathbf{S}$ & $\mathbf{S}$ & $\mathbf{S}$ & $\mathbf{R}$ & $\mathbf{S}$ & $\mathbf{S}$ & $\mathbf{S}$ & $\mathbf{S}$ & $\mathbf{R}$ \\
\hline
\end{tabular}

$\mathrm{S}=$ Sensitive $\mathbf{I}=$ Intermediate $\mathbf{R}=$ Resistant.

Amp= Ampicillin, AMX= Amoxicillin, AMX-Na= Amoxicillin-Naladixic acid,Cip= Ciprofloxacin,Imp= Imipenem, CTX = Cefotaxime, $\mathrm{CRO}=$ Cefterixon, Tri $=$ Trimethoprime $\quad \mathrm{CF}=\quad$ Cephalothin, $\quad \mathrm{TE}=$ Tetracycline, $\mathrm{C}=$ Chloramphenicol, Nor= Norfloxacin, and $\mathrm{E}=$ Erythromycin

Antimicrobial activity of the Allium cepa (onion) extracts on Aeromonashydrophila isolates:-

Among the three types of white onion extracts were tested,only hot - water extract showed antibacterial activity. Different Aeromonashydrophila isolates responded differently to the white onion hot - water extract at different concentrations.

The results of the antibacterial activity of onion extracts against Aeromonashydrophila was listed in table-5 and figure-2.

Purple onion extracts did not show any inhibitory effect on the growth of Aeromonashydrophila isolates. The results were listed in table -6 . The widest zones of inhibition was obtained with the $500 \mu \mathrm{g} / \mathrm{ml}$ concentration of hot - water onion extract. These differences in the zones of inhibition may be directly related to the susceptibility of each isolates to the onions extracts.

Table 5:-Antibacterial activity of different concentrations of Allium cepa (white onion) extracts, cold - water extract, hot-water extract and ethanolicextract on Aeromonashydrophilaby cup-plate diffusion method.

\begin{tabular}{|c|c|c|c|c|c|c|c|c|c|c|c|c|c|c|c|}
\hline \multirow{3}{*}{$\begin{array}{l}\text { No. of } \\
\text { isolates }\end{array}$} & \multicolumn{15}{|c|}{ Diameter of inhibition zone (in $\mathrm{mm}$ ) against various concentrations of onion extracts } \\
\hline & \multicolumn{5}{|c|}{$\begin{array}{c}\text { Conc. Of extracts } \mu \text { glml } \\
\text { cold - water extract }\end{array}$} & \multicolumn{5}{|c|}{$\begin{array}{c}\text { Conc. Of extracts } \mu \text { glml } \\
\text { hot-water extract }\end{array}$} & \multicolumn{5}{|c|}{$\begin{array}{c}\text { Conc. Of extracts } \mu \text { glml } \\
\text { ethanolic extract }\end{array}$} \\
\hline & 100 & 200 & 300 & 400 & 500 & 100 & 200 & 300 & 400 & 500 & 100 & 200 & 300 & 400 & 500 \\
\hline 1 & $\mathbf{0}$ & $\mathbf{0}$ & $\mathbf{0}$ & $\mathbf{0}$ & $\mathbf{0}$ & $\mathbf{0}$ & (0 & $\mathbf{0}$ & ( & $\mathbf{0}$ & $\mathbf{0}$ & $\mathbf{0}$ & 0 & 0 & $\mathbf{0}$ \\
\hline 2 & $\mathbf{0}$ & $\mathbf{0}$ & $\mathbf{0}$ & $\mathbf{0}$ & $\mathbf{0}$ & 8 & 8 & 10 & 12 & 12 & $\mathbf{0}$ & $\mathbf{0}$ & $\mathbf{0}$ & $\mathbf{0}$ & 0 \\
\hline 3 & $\mathbf{0}$ & $\mathbf{0}$ & (0) & (0) & $\mathbf{0}$ & (0) & (0) & $\mathbf{0}$ & (0) & (0) & (0) & (0 & $\mathbf{0}$ & (0 & $\mathbf{0}$ \\
\hline
\end{tabular}




\begin{tabular}{|c|c|c|c|c|c|c|c|c|c|c|c|c|c|c|c|}
\hline $\mathbf{4}$ & $\mathbf{0}$ & $\mathbf{0}$ & $\mathbf{0}$ & $\mathbf{0}$ & $\mathbf{0}$ & $\mathbf{0}$ & $\mathbf{0}$ & $\mathbf{0}$ & $\mathbf{0}$ & $\mathbf{0}$ & $\mathbf{0}$ & $\mathbf{0}$ & $\mathbf{0}$ & $\mathbf{0}$ & $\mathbf{0}$ \\
\hline $\mathbf{5}$ & $\mathbf{0}$ & $\mathbf{0}$ & $\mathbf{0}$ & $\mathbf{0}$ & $\mathbf{0}$ & $\mathbf{1 0}$ & $\mathbf{1 0}$ & $\mathbf{1 2}$ & $\mathbf{1 4}$ & $\mathbf{1 4}$ & $\mathbf{0}$ & $\mathbf{0}$ & $\mathbf{0}$ & $\mathbf{0}$ & $\mathbf{0}$ \\
\hline $\mathbf{6}$ & $\mathbf{0}$ & $\mathbf{0}$ & $\mathbf{0}$ & $\mathbf{0}$ & $\mathbf{0}$ & $\mathbf{0}$ & $\mathbf{0}$ & $\mathbf{0}$ & $\mathbf{0}$ & $\mathbf{0}$ & $\mathbf{0}$ & $\mathbf{0}$ & $\mathbf{0}$ & $\mathbf{0}$ & $\mathbf{0}$ \\
\hline $\mathbf{7}$ & $\mathbf{0}$ & $\mathbf{0}$ & $\mathbf{0}$ & $\mathbf{0}$ & $\mathbf{0}$ & $\mathbf{6}$ & $\mathbf{6}$ & $\mathbf{6}$ & $\mathbf{8}$ & $\mathbf{1 0}$ & $\mathbf{0}$ & $\mathbf{0}$ & $\mathbf{0}$ & $\mathbf{0}$ & $\mathbf{0}$ \\
\hline $\mathbf{8}$ & $\mathbf{0}$ & $\mathbf{0}$ & $\mathbf{0}$ & $\mathbf{0}$ & $\mathbf{0}$ & $\mathbf{0}$ & $\mathbf{0}$ & $\mathbf{0}$ & $\mathbf{0}$ & $\mathbf{0}$ & $\mathbf{0}$ & $\mathbf{0}$ & $\mathbf{0}$ & $\mathbf{0}$ & $\mathbf{0}$ \\
\hline $\mathbf{9}$ & $\mathbf{0}$ & $\mathbf{0}$ & $\mathbf{0}$ & $\mathbf{0}$ & $\mathbf{0}$ & $\mathbf{1 1}$ & $\mathbf{1 1}$ & $\mathbf{1 3}$ & $\mathbf{1 3}$ & $\mathbf{1 3}$ & $\mathbf{0}$ & $\mathbf{0}$ & $\mathbf{0}$ & $\mathbf{0}$ & $\mathbf{0}$ \\
\hline $\mathbf{1 0}$ & $\mathbf{0}$ & $\mathbf{0}$ & $\mathbf{0}$ & $\mathbf{0}$ & $\mathbf{0}$ & $\mathbf{8}$ & $\mathbf{8}$ & $\mathbf{9}$ & $\mathbf{9}$ & $\mathbf{1 0}$ & $\mathbf{0}$ & $\mathbf{0}$ & $\mathbf{0}$ & $\mathbf{0}$ & $\mathbf{0}$ \\
\hline $\mathbf{1 1}$ & $\mathbf{0}$ & $\mathbf{0}$ & $\mathbf{0}$ & $\mathbf{0}$ & $\mathbf{0}$ & $\mathbf{9}$ & $\mathbf{9}$ & $\mathbf{9}$ & $\mathbf{9}$ & $\mathbf{9}$ & $\mathbf{0}$ & $\mathbf{0}$ & $\mathbf{0}$ & $\mathbf{0}$ & $\mathbf{0}$ \\
\hline $\mathbf{1 2}$ & $\mathbf{0}$ & $\mathbf{0}$ & $\mathbf{0}$ & $\mathbf{0}$ & $\mathbf{0}$ & $\mathbf{9}$ & $\mathbf{9}$ & $\mathbf{9}$ & $\mathbf{9}$ & $\mathbf{9}$ & $\mathbf{0}$ & $\mathbf{0}$ & $\mathbf{0}$ & $\mathbf{0}$ & $\mathbf{0}$ \\
\hline $\mathbf{1 3}$ & $\mathbf{0}$ & $\mathbf{0}$ & $\mathbf{0}$ & $\mathbf{0}$ & $\mathbf{0}$ & $\mathbf{0}$ & $\mathbf{0}$ & $\mathbf{0}$ & $\mathbf{0}$ & $\mathbf{0}$ & $\mathbf{0}$ & $\mathbf{0}$ & $\mathbf{0}$ & $\mathbf{0}$ & $\mathbf{0}$ \\
\hline $\mathbf{1 4}$ & $\mathbf{0}$ & $\mathbf{0}$ & $\mathbf{0}$ & $\mathbf{0}$ & $\mathbf{0}$ & $\mathbf{1 0}$ & $\mathbf{1 0}$ & $\mathbf{1 2}$ & $\mathbf{1 4}$ & $\mathbf{1 5}$ & $\mathbf{0}$ & $\mathbf{0}$ & $\mathbf{0}$ & $\mathbf{0}$ & $\mathbf{0}$ \\
\hline $\mathbf{1 5}$ & $\mathbf{0}$ & $\mathbf{0}$ & $\mathbf{0}$ & $\mathbf{0}$ & $\mathbf{0}$ & $\mathbf{8}$ & $\mathbf{8}$ & $\mathbf{1 0}$ & $\mathbf{1 0}$ & $\mathbf{1 0}$ & $\mathbf{0}$ & $\mathbf{0}$ & $\mathbf{0}$ & $\mathbf{0}$ & $\mathbf{0}$ \\
\hline $\mathbf{1 6}$ & $\mathbf{0}$ & $\mathbf{0}$ & $\mathbf{0}$ & $\mathbf{0}$ & $\mathbf{0}$ & $\mathbf{8}$ & $\mathbf{8}$ & $\mathbf{1 0}$ & $\mathbf{1 0}$ & $\mathbf{1 1}$ & $\mathbf{0}$ & $\mathbf{0}$ & $\mathbf{0}$ & $\mathbf{0}$ & $\mathbf{0}$ \\
\hline $\mathbf{1 7}$ & $\mathbf{0}$ & $\mathbf{0}$ & $\mathbf{0}$ & $\mathbf{0}$ & $\mathbf{0}$ & $\mathbf{9}$ & $\mathbf{9}$ & $\mathbf{1 3}$ & $\mathbf{1 5}$ & $\mathbf{1 5}$ & $\mathbf{0}$ & $\mathbf{0}$ & $\mathbf{0}$ & $\mathbf{0}$ & $\mathbf{0}$ \\
\hline $\mathbf{1 8}$ & $\mathbf{0}$ & $\mathbf{0}$ & $\mathbf{0}$ & $\mathbf{0}$ & $\mathbf{0}$ & $\mathbf{1 0}$ & $\mathbf{1 0}$ & $\mathbf{1 0}$ & $\mathbf{1 0}$ & $\mathbf{1 0}$ & $\mathbf{0}$ & $\mathbf{0}$ & $\mathbf{0}$ & $\mathbf{0}$ & $\mathbf{0}$ \\
\hline $\mathbf{1 9}$ & $\mathbf{0}$ & $\mathbf{0}$ & $\mathbf{0}$ & $\mathbf{0}$ & $\mathbf{0}$ & $\mathbf{0}$ & $\mathbf{0}$ & $\mathbf{0}$ & $\mathbf{0}$ & $\mathbf{0}$ & $\mathbf{0}$ & $\mathbf{0}$ & $\mathbf{0}$ & $\mathbf{0}$ & $\mathbf{0}$ \\
\hline $\mathbf{2 0}$ & $\mathbf{0}$ & $\mathbf{0}$ & $\mathbf{0}$ & $\mathbf{0}$ & $\mathbf{0}$ & $\mathbf{0}$ & $\mathbf{0}$ & $\mathbf{0}$ & $\mathbf{0}$ & $\mathbf{0}$ & $\mathbf{0}$ & $\mathbf{0}$ & $\mathbf{0}$ & $\mathbf{0}$ & $\mathbf{0}$ \\
\hline
\end{tabular}

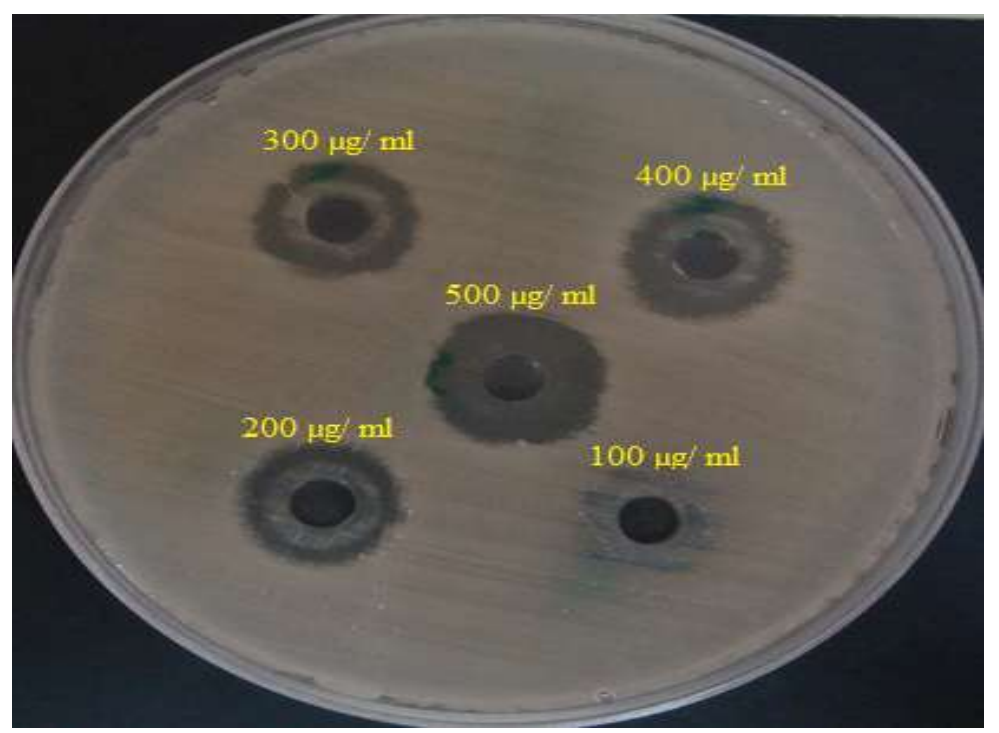

Figure-2:-Antibacterial activity of white onion extracts, hot - water extract

In Induet al., (3) study, the various concentrations of onion extract failed to inhibit the growth of all tested organisms (E. coli, Listeria monocytogenes, Salmonella, andAeromonashydrophila). The differences may be due to a difference in the variety of the onion used in this study.

Table 6:-Antibacterial activity of different concentrations of Allium cepa (purple onion) extracts, cold - water extract, hot-water extract and ethanolic extract on Aeromonashydrophilaby cup-plate diffusion method.

\begin{tabular}{|c|c|c|c|c|c|c|c|c|c|c|c|c|c|c|c|}
\hline \multirow{3}{*}{$\begin{array}{l}\text { No. of } \\
\text { isolates }\end{array}$} & \multicolumn{15}{|c|}{ Diameter of inhibition zone (in $\mathrm{mm}$ ) against various concentrations of onion extracts } \\
\hline & \multicolumn{5}{|c|}{$\begin{array}{c}\text { Conc. Of extracts } \mu \text { glml } \\
\text { cold - water extract }\end{array}$} & \multicolumn{5}{|c|}{$\begin{array}{c}\text { Conc. Of extracts } \mu \text { glml } \\
\text { hot-water extract }\end{array}$} & \multicolumn{5}{|c|}{$\begin{array}{c}\text { Conc. Of extracts } \mu g \operatorname{lml} \\
\text { ethanolic extract }\end{array}$} \\
\hline & 100 & 200 & 300 & 400 & 500 & 100 & 200 & 300 & 400 & 500 & 100 & 200 & 300 & 400 & 500 \\
\hline 1 & $\mathbf{0}$ & $\mathbf{0}$ & $\mathbf{0}$ & $\mathbf{0}$ & 0 & 0 & 0 & 0 & 0 & 0 & 0 & 0 & 0 & $\mathbf{0}$ & 0 \\
\hline 2 & $\mathbf{0}$ & $\mathbf{0}$ & $\mathbf{0}$ & $\mathbf{0}$ & $\mathbf{0}$ & $\mathbf{0}$ & $\mathbf{0}$ & $\mathbf{0}$ & $\mathbf{0}$ & $\mathbf{0}$ & $\mathbf{0}$ & $\mathbf{0}$ & $\mathbf{0}$ & $\mathbf{0}$ & 0 \\
\hline 3 & 0 & 0 & 0 & 0 & 0 & 0 & $\mathbf{0}$ & 0 & 0 & ( & 0 & 0 & 0 & 0 & 0 \\
\hline 4 & $\mathbf{0}$ & $\mathbf{0}$ & $\mathbf{0}$ & 0 & $\mathbf{0}$ & 0 & 0 & (0) & (0) & ( & $\mathbf{0}$ & $\mathbf{0}$ & (0) & $\mathbf{0}$ & $\mathbf{0}$ \\
\hline 5 & $\mathbf{0}$ & $\mathbf{0}$ & $\mathbf{0}$ & $\mathbf{0}$ & $\mathbf{0}$ & $\mathbf{0}$ & $\mathbf{0}$ & $\mathbf{0}$ & $\mathbf{0}$ & $\mathbf{0}$ & $\mathbf{0}$ & $\mathbf{0}$ & $\mathbf{0}$ & $\mathbf{0}$ & $\mathbf{0}$ \\
\hline 6 & 0 & $\mathbf{0}$ & $\mathbf{0}$ & $\mathbf{0}$ & $\mathbf{0}$ & $\mathbf{0}$ & $\mathbf{0}$ & $\mathbf{0}$ & $\mathbf{0}$ & $\mathbf{0}$ & $\mathbf{0}$ & $\mathbf{0}$ & (0 & $\mathbf{0}$ & $\mathbf{0}$ \\
\hline 7 & ( & (0) & (0) & (0) & (0 & (0 & $\mathbf{0}$ & $\mathbf{0}$ & (0 & $\mathbf{0}$ & (0) & $\mathbf{0}$ & (0 & (0) & $\mathbf{0}$ \\
\hline
\end{tabular}




\begin{tabular}{|c|c|c|c|c|c|c|c|c|c|c|c|c|c|c|c|}
\hline $\mathbf{8}$ & $\mathbf{0}$ & $\mathbf{0}$ & $\mathbf{0}$ & $\mathbf{0}$ & $\mathbf{0}$ & $\mathbf{0}$ & $\mathbf{0}$ & $\mathbf{0}$ & $\mathbf{0}$ & $\mathbf{0}$ & $\mathbf{0}$ & $\mathbf{0}$ & $\mathbf{0}$ & $\mathbf{0}$ & $\mathbf{0}$ \\
\hline $\mathbf{9}$ & $\mathbf{0}$ & $\mathbf{0}$ & $\mathbf{0}$ & $\mathbf{0}$ & $\mathbf{0}$ & $\mathbf{0}$ & $\mathbf{0}$ & $\mathbf{0}$ & $\mathbf{0}$ & $\mathbf{0}$ & $\mathbf{0}$ & $\mathbf{0}$ & $\mathbf{0}$ & $\mathbf{0}$ & $\mathbf{0}$ \\
\hline $\mathbf{1 0}$ & $\mathbf{0}$ & $\mathbf{0}$ & $\mathbf{0}$ & $\mathbf{0}$ & $\mathbf{0}$ & $\mathbf{0}$ & $\mathbf{0}$ & $\mathbf{0}$ & $\mathbf{0}$ & $\mathbf{0}$ & $\mathbf{0}$ & $\mathbf{0}$ & $\mathbf{0}$ & $\mathbf{0}$ & $\mathbf{0}$ \\
\hline $\mathbf{1 1}$ & $\mathbf{0}$ & $\mathbf{0}$ & $\mathbf{0}$ & $\mathbf{0}$ & $\mathbf{0}$ & $\mathbf{0}$ & $\mathbf{0}$ & $\mathbf{0}$ & $\mathbf{0}$ & $\mathbf{0}$ & $\mathbf{0}$ & $\mathbf{0}$ & $\mathbf{0}$ & $\mathbf{0}$ & $\mathbf{0}$ \\
\hline $\mathbf{1 2}$ & $\mathbf{0}$ & $\mathbf{0}$ & $\mathbf{0}$ & $\mathbf{0}$ & $\mathbf{0}$ & $\mathbf{0}$ & $\mathbf{0}$ & $\mathbf{0}$ & $\mathbf{0}$ & $\mathbf{0}$ & $\mathbf{0}$ & $\mathbf{0}$ & $\mathbf{0}$ & $\mathbf{0}$ & $\mathbf{0}$ \\
\hline $\mathbf{1 3}$ & $\mathbf{0}$ & $\mathbf{0}$ & $\mathbf{0}$ & $\mathbf{0}$ & $\mathbf{0}$ & $\mathbf{0}$ & $\mathbf{0}$ & $\mathbf{0}$ & $\mathbf{0}$ & $\mathbf{0}$ & $\mathbf{0}$ & $\mathbf{0}$ & $\mathbf{0}$ & $\mathbf{0}$ & $\mathbf{0}$ \\
\hline $\mathbf{1 4}$ & $\mathbf{0}$ & $\mathbf{0}$ & $\mathbf{0}$ & $\mathbf{0}$ & $\mathbf{0}$ & $\mathbf{0}$ & $\mathbf{0}$ & $\mathbf{0}$ & $\mathbf{0}$ & $\mathbf{0}$ & $\mathbf{0}$ & $\mathbf{0}$ & $\mathbf{0}$ & $\mathbf{0}$ & $\mathbf{0}$ \\
\hline $\mathbf{1 5}$ & $\mathbf{0}$ & $\mathbf{0}$ & $\mathbf{0}$ & $\mathbf{0}$ & $\mathbf{0}$ & $\mathbf{0}$ & $\mathbf{0}$ & $\mathbf{0}$ & $\mathbf{0}$ & $\mathbf{0}$ & $\mathbf{0}$ & $\mathbf{0}$ & $\mathbf{0}$ & $\mathbf{0}$ & $\mathbf{0}$ \\
\hline $\mathbf{1 6}$ & $\mathbf{0}$ & $\mathbf{0}$ & $\mathbf{0}$ & $\mathbf{0}$ & $\mathbf{0}$ & $\mathbf{0}$ & $\mathbf{0}$ & $\mathbf{0}$ & $\mathbf{0}$ & $\mathbf{0}$ & $\mathbf{0}$ & $\mathbf{0}$ & $\mathbf{0}$ & $\mathbf{0}$ & $\mathbf{0}$ \\
\hline $\mathbf{1 7}$ & $\mathbf{0}$ & $\mathbf{0}$ & $\mathbf{0}$ & $\mathbf{0}$ & $\mathbf{0}$ & $\mathbf{0}$ & $\mathbf{0}$ & $\mathbf{0}$ & $\mathbf{0}$ & $\mathbf{0}$ & $\mathbf{0}$ & $\mathbf{0}$ & $\mathbf{0}$ & $\mathbf{0}$ & $\mathbf{0}$ \\
\hline $\mathbf{1 8}$ & $\mathbf{0}$ & $\mathbf{0}$ & $\mathbf{0}$ & $\mathbf{0}$ & $\mathbf{0}$ & $\mathbf{0}$ & $\mathbf{0}$ & $\mathbf{0}$ & $\mathbf{0}$ & $\mathbf{0}$ & $\mathbf{0}$ & $\mathbf{0}$ & $\mathbf{0}$ & $\mathbf{0}$ & $\mathbf{0}$ \\
\hline 19 & $\mathbf{0}$ & $\mathbf{0}$ & $\mathbf{0}$ & $\mathbf{0}$ & $\mathbf{0}$ & $\mathbf{0}$ & $\mathbf{0}$ & $\mathbf{0}$ & $\mathbf{0}$ & $\mathbf{0}$ & $\mathbf{0}$ & $\mathbf{0}$ & $\mathbf{0}$ & $\mathbf{0}$ & $\mathbf{0}$ \\
\hline $\mathbf{2 0}$ & $\mathbf{0}$ & $\mathbf{0}$ & $\mathbf{0}$ & $\mathbf{0}$ & $\mathbf{0}$ & $\mathbf{0}$ & $\mathbf{0}$ & $\mathbf{0}$ & $\mathbf{0}$ & $\mathbf{0}$ & $\mathbf{0}$ & $\mathbf{0}$ & $\mathbf{0}$ & $\mathbf{0}$ & $\mathbf{0}$ \\
\hline
\end{tabular}

\section{Conclusions:-}

1. Isolation of Aeromonashydrophila was the highest in age range $(0-2)$ years old .

2. Ciproflaxacin, norfloxacin, chloramphinicol, tetracycline, cefterixone, cefotaxime and imipenem were the most effective antibiotics against local isolates of Aeromonashydrophila.

3. Only hot - water extract of white Allium cepa (onion) showed antibacterial activity against Aeromonashydrophila isolates .

\section{References:-}

1. Trower, C. J.; ABO, S.; Majeed, K. N.; andVonitzstein, M. (2000). Production of an enterotoxin by a gastroenteritis associated Aeromonas strains. J. Med. Microbiol., 49:121-126.

2. Deodhar, L. P.; Saraswathi,K. andVarudkar, A. (1991). Aeromonas spp. and their association with human diarrheal disease. J. Clin. Microbiol., 29:853-856.

3. Indu, M.N.; Hatha, A.A.M.; Abirosh, C.; Harsha, U. andVivekanandan, G.(2006). Antibacterial Activity of Some of the South - Indian Spices Against Serotypes of Escherichia coli, Salmonella, Listeria monocytogenes and Aeromonashydrophila. Braz. J. of Microbiol. 37: 153-158.

4. Tsai, G. and Chen, T. (1996). Incidence and toxigenicity of Aeromonashydrophila in seafood. Int. J. Food. Microbiol. 31(1-2): 121-131.

5. Forbes, B. A.;Sahm, D. F. andWeissfeld, A. S. (1998). Vibrio, Aeromonas, Plesiomonasshigelloides, \&Chromobacteriumviolaceum, p.488-500. In: B. A. Forbes;D. F. Sahm; A. S. Weissfeld (eds.), Bailey \& Scott's Diagnostic Microbiology, 10th ed., Mosbey-year Book-Inc. Baltimor. Boston.

6. Ciprian, R.C. (2001). Aeromonashydrophila and motile Aeromonad septicemias of fish.Rev.FishDis.Leaflet.68:1-20.

7. Baron, E. J. and Finegold, S. M. (1990). Bailey \& Scotts Diagnostic Microbiology. 8th (ed.). Mosby, Comp. Missouri.

8. National Committee for clinical laboratory standards (NCCLs). (2002) Preformance standards for antimicrobial susceptibility testing. Villanova P.A.U.S.A.

9. Azu, N.C. and Onyeagba, R.A. (2007). Antimicrobial Properties Of Extracts Of Allium cepa (Onions) And Zingiberofficinale (Ginger) On Escherichia coli, Salmonella typhi And Bacillus subtilis . The Internet Journal of Tropical Medicine. 3 (2): 14-19.

10. Robinson, J.; Burke, V.; Worthy, P. J.; Beaman, J. and Wagener, L. (1984). Media for isolation of Aeromonas spp. from feaces. J. Med. Microbiol. 18: 405-411.

11. Kay, B.; Guerrero, C. and Sack, R. (1985). Media for the isolation of Aeromonashydrophila. J. Clin. Microbiol. 22(5): 888-890.

12. Aslani1, M. M and Alikhani, M.Y.(2004)The Role of Aeromonashydrophilain Diarrhea Iranian .J. Pub. Health. 33(3): 54-59.

13. Juan, H. J.; Tang, R.; Wu, T.; and Yu,K.(2000). Isolation of Aeromonashydrophila in children with diarrhea. J.Microbiol. Imm. Inf. 33:115 - 117.

14. Kannan, S.; Chattopadhyay, U.; Pal, D.; Shimada, T.; Takeda, Y.; Bhattacharya, S. and Ananthanarayanan, P. (2001). Isolation and identification of Aeromonasfrom patients with acute diarrhoea in Kolkata, India. Ind. J. Med. Microbiol. 19 (4): 1-11. 
15. Obi, C. L. and Bessong, P. O. (2002). Diarrhoeagenic bacterial pathogens in HIV-positive patients with diarrhoea in rural communities of Limpopo Province, South Africa. J. Health. Popul. Nutr. 20(3): 230-234.

16. Ram, S.; Khurana, S.; Sharma, S. and Vadehra, D. (1991). Aeromonas- associated diarrhoea in north India. J. Diarrhoeal. Dis. Res. 9(1): 23-28.

17. Mikhail, I.; Fox, E.; Haberberger, R.; Ahmed, M. and Abbatte, E. (1990). Epidemiology of bacterial pathagens associated with infectious diarrhea in Djibouti. J. Clin. Microbiol. 28(5): 956-961.

18. 